\title{
Fluid-Structure Coupled Analysis of a Fabric-Covered Airfoil
}

\author{
Jae-Sunbe Bae, Soo-Yong Lee, Hark-Bong Kim, Jung-Sun Park \\ KoreaAerospace University \\ 200-1, Hwajeon-dong, Deogynag-gu, Goyang-city, Gyeonggi-do, South Korea \\ jsbae@kau.ac.kr; leesy@kau.ac.kr; hbkim@kau.ac.kr; jungsun@kau.ac.kr
}

\section{Extended Abstract}

Wind turbines (WTs) rapidly become bigger than bigger but conventional WT blade designs have still used fiberglass. Though fiberglass is cheaper than advanced materials like carbon composites it is heavy and its manufacturing process is extremely hands-on time intensive. This conventional WT blade design may not be good for the development of advanced WT blades. A new approach to design, manufacture, and install WT blades will be necessary in the near future. The use of architectural fabrics could change the conventional design of the WT blades [1] and could reduce the manufacturing costs of very large blade manufacturing $25 \%$ to $40 \%$. This fabric-covered wind turbine blade is composed of spars, ribs and covering fabrics similar to the truss structure of the aircraft wings. In this concept, the front and rear spars sustain all of the loads like bending moments of a wing. The fabric-skin is mainly subjected to torque and keeps the airfoil shape. This fabric skin acts like a membrane and is deformed by the pressure acting on it. If it deforms the pressure distribution acting on it is also changed and consequently the fabric skin deforms again. Finally, due to the fabric skin deformation, the aerodynamic characteristics of the blade and wing are changed and the aeroelastic characteristics of the fabric skin should be investigated.

In the present study, the aerodynamic deformations and aeroelastic characteristics of the fabric skin are studied. The membrane analysis of the fabric skin is performed for its structural modelling and the CFD (computational fluid dynamics) analysis of the airfoil section is performed by using ANSYS. The present membrane analysis results and CFD results are verified by comparing the reference results. Finally, the fluid-structure coupled analsys of the fabric skin are performed and its aerodynamic deformations, deformed pressure distributions, and aeroelastic characteristics are investigated.

\section{Acknowledgements}

This work was supported by the Ministry of Trade, Industry \& Energy (MOTIE, Korea) under the Industrial Technological Innovation Program of the Korea Evaluation Institute of Industrial Technology (KEIT), and the New \& Renewable Energy Core Technology Program of the Korea Institute of Energy Technology Evaluation and Planning (KETEP). Also, this work was partially supported by UCONSYSTEM Co., Ltd.

\section{References}

[1] http://www.thevoltreport.com/fabric-wind-turbine-blades-for-the-new-new-renewable-power/ 